\title{
Role of Genetic Variants and Gene Expression in the Susceptibility and Severity of COVID-19
}

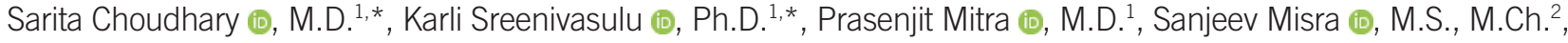 \\ and Praveen Sharma $\mathbb{0}$, Ph.D. ${ }^{1}$ \\ Departments of ${ }^{1}$ Biochemistry and ${ }^{2}$ Surgical Oncology, All India Institute of Medical Sciences, Jodhpur, India
}

\begin{abstract}
Since its first report in December 2019, coronavirus disease 2019 (COVID-19), caused by severe acute respiratory syndrome coronavirus 2 (SARS-CoV-2), has rapidly emerged as a pandemic affecting nearly all countries worldwide. As the COVID-19 pandemic progresses, the need to identify genetic risk factors for susceptibility to this serious illness has emerged. Host genetic factors, along with other risk factors may help determine susceptibility to respiratory tract infections. It is hypothesized that the ACE2 gene, encoding angiotensin-converting enzyme 2 (ACE2), is a genetic risk factor for SARS-CoV-2 infection and is required by the virus to enter cells. Together with ACE2, transmembrane protease serine 2 (TMPRSS2) and dipeptidyl peptidase-4 (DPP4) also play an important role in disease severity. Evaluating the role of genetic variants in determining the direction of respiratory infections will help identify potential drug target candidates for further study in COVID-19 patients. We have summarized the latest reports demonstrating that ACE2 variants, their expression, and epigenetic factors may influence an individual's susceptibility to SARSCoV-2 infection and disease outcome.
\end{abstract}

Key Words: Angiotensin-converting enzyme 2 (ACE2) variants, Transmembrane protease, Serine 2 (TMPRSS2), Epigenetics, COVID-19, SARS-CoV-2 infection

\author{
Received: July 6, 2020 \\ Revision received: July 20, 2020 \\ Accepted: September 22, 2020
}

\section{Corresponding author: \\ Prasenjit Mitra, M.D., MRSB, FACSC Department of Biochemistry, All India Institute of Medical Sciences, Basni Industrial Area Phase-2, Jodhpur 342005, Rajasthan, India \\ Tel: +91-8107835666 \\ E-mail: prasy4u@gmail.com; \\ mitrap@aiimsjodhpur.edu.in \\ *These authors contributed equally to this study.}

\section{(i) (5)}

(c) Korean Society for Laboratory Medicine

This is an Open Access article distributed under the terms of the Creative Commons Attribution Non-Commercial License (https://creativecommons.org/licenses/by-nc/4.0) which permits unrestricted non-commercial use, distribution, and reproduction in any medium, provided the original work is properly cited.

\section{INTRODUCTION}

Coronavirus disease 2019 (COVID-19) emerged in Wuhan city, China, in December 2019. On January 12, 2020, the World Health Organization (WHO) named the causative virus as novel coronavirus (nCov); the virus was renamed on February 11, 2020, as severe acute respiratory syndrome coronavirus 2 (SARS-CoV-2) by the international virus classification commission [1]. During the initial days of the epidemic, various countries followed and implemented different testing strategies, based on the accessibility of diagnostic methods [2, 3]. COVID-19 causes respiratory illness that may range from a mild viral pneumonia to acute respiratory distress syndrome (ARDS) leading to multi-organ failure. The elderly population and individuals suffering from chronic illness have to be extra cautious, as the mortality rate is considerably high among these groups [4]. The massive spread of the virus across countries within a few months of onset led the WHO to declare COVID-19 a pandemic. As of July 19, 2020, the total number of cases and deaths worldwide stood at 14,043,176 and 597,583, respectively [5].

SARS-CoV-2 is transmitted to lung epithelial cells through aerosols. Various immune cells in the body may also augment the infection; peripheral neutrophilia and lymphocytopenia significantly induce the immune response and cause deleterious ARDS, multi-organ dysfunction, and finally the death in some patients. Additionally, a sudden surge of pro-inflammatory cyto- 
kines may lead to fatal outcomes, indicating that cytokines are crucial in the pathophysiology of COVID-19 [6].

These findings have raised the question of whether genetic or epigenetic variation can be used to identify symptom susceptibility or severity in this disease. A review of a number of studies has suggested that genetic factors along with other risk factors can determine an individual's susceptibility to respiratory tract infections [7]. As angiotensin-converting enzyme 2 (ACE2) receptor on host cells acts as an entry point for SARS-CoV-2, it is hypothesized that the ACE2 gene constitutes a genetic risk factor for SARS-CoV-2 infection [8]. In this regard, ACE2 variants previously reported to be associated with other disorders, such as hypertension and other cardiovascular diseases, pose promising genetic factor candidates [9, 10]. Moreover, variation in the expression of ACE2 and associated epigenetic factors may play a significant role in determining an individual's susceptibility to COVID-19. This review provides an update on the possible role of genetic variants, gene expression, and epigenetic factors in the pathophysiology of COVID-19.

\section{Coronaviruses}

Coronaviruses are members of the enveloped single-stranded RNA viruses that cause respiratory, enteric, and cardiovascular diseases in humans and animals. Various coronaviruses, such as NL63, 229E, and OC43, have been found to be altered for human spread [11]. Many different types of coronaviruses have arisen from various bat species in China; these are mostly direct SARS-CoV ancestors [12]. Initially, the SARS-CoV that triggered an epidemic in humans originated from Chinese horseshoe bats, Rhinolophus, which originally adapted to the wild Himalayan palm civet [13]. The novel betacoronavirus (SARS-CoV-2) that causes COVID-19 shares $79.5 \%$ nucleotide identity with SARS-CoV [14].

During earlier SARS outbreaks, studies demonstrated, in an autopsy series using culture techniques, viral isolation, and in situ hybridization, that SARS-CoV exists in pneumocytes [15, 16]. The existence of coronavirus-like particles and viral particles in pneumocytes has also been revealed by transmission electron microscopy [17]. Further evidence indicates that ACE2 serves as the functional receptor for SARS-CoV, suggesting that alveolar pneumocytes are a potential entry point for SARS-CoV in the lung [18]. ACE2 staining was strongly positive for type I and II pneumocytes, whereas weak staining was observed in bronchial epithelial cells.

\section{ACE2 structure and function}

ACE2 is an ACE homologue discovered in 2000. The ACE2 gene is found on chromosome Xp22 and comprises 18 exons [19]. ACE2 has only one catalytic domain, acts as a zinc metallopeptidase, and its distinct and counterbalancing functions in the renin-angiotensin system are characterized by different substrate specificities. ACE2 functions as a simple carboxy peptidase; it hydrolyzes angiotensin I (Ang I) to Ang 1-9 and Ang II to Ang 1-7 [20]. ACE2 has higher affinity to Ang II than to Ang I; its catalytic activity with Ang II is 400-fold higher than with Ang I [21].

ACE2 has multiple roles including catalytic activities with specific substrates; it acts as a negative regulator of the renin-aldosterone system, BO AT1 amino acid transporter, and receptor for coronaviruses (SARS-CoV) [22]. ACE2 restricts the adverse effects of Ang II on profibrotic and vasoconstrictors. The hydrolysis of Ang II into Ang 1-7 decreases oxidative stress, and Ang 1-7 has counter regulatory mechanisms including antifibrotic and vasodilatory actions. Consequently, ACE2 disturbance leads to elevated Ang II levels and reduced heart function [22]. ACE2 is active in several tissues and is widespread in the lungs, kidneys, heart, and testis. Low levels of ACE2 mRNA expression have been associated with hypertension and heart failure and reduced levels of cardiac ACE2 have been reported in hypertension and diabetic heart disease. These multiple physiological roles of ACE2 have been hijacked by SARS-CoV-2 to serve as a receptor, resulting in COVID-19 [23].

\section{ACE2 as SARS-CoV-2 primary receptor}

Previously, Li, et al. [24] reported that ACE2 is a key receptor for the HCoV-NL63 coronavirus, which caused the SARS outbreak. In addition to SARS-CoV-2, the ACE2 receptor has been linked with MERS and Hartnup disorder [25]. Cellular ACE2 expression levels differ among individuals. A recent study reported that the distribution of ACE2 expression is organ-specific [26].

\section{ACE2 variation and SARS-CoV-2 receptor binding domain} ACE2 utilization by SARS-CoV-2 can be explored to gain insights into the mechanism of restricting SARS-CoV-2 entry into host cells. Although ACE2 is expressed in most vertebrates, SARSCoV-2 cannot always utilize it as a receptor. The ability of SARSCoV-2 to utilize ACE2 was predicted by observing few essential single amino acid $(A A)$ variant sites, which were found to be important for SARS-CoV-2 [27]. Recent reports showed that SARSCoV-2 can utilize ACE2 expressed in humans, Chinese horseshoe bats, swine, and civets, but not that expressed in mouse [28]. In another study, Qiu, et al. [29] evaluated ACE2 homology 
among five different species and observed 11 discrete mouse AA residues (T20, Y83, S218, A246, K353, P426, T593, N636, A714, R716, and A774), which are conserved in other ACEs. Although the binding of human ACE2 and the receptor-binding domain (RBD) of the SARS-CoV-2 spike (S) protein is quite complex, out of the 11 conserved AA residues, only three (T20, Y83, and K353) are located near the interface and thereby may facilitate binding [29]. Consequently, AA residues considered important for SARS-CoV receptor binding may also be critical for SARS-CoV-2 as their RBD AA sequences are $74.6 \%$ identical. Of the previously reported important sites (K31, Y41, K68, Y83, K353, D355, R357, and M383) [30], substitutions in K68 and M383 slightly inhibit SARS-CoV binding, while those in K31D, K353 H/A/D, D355A, Y41A, Y83F, and R357A strongly inhibit the SARS-CoV binding and could also abolish the interaction [31]. Using these substitution methods, Qiu, et al. [29] identified nine critical AA sites and evaluated various types of ACE2 utilization by SARS-CoV-2.

\section{Structural basis for SARS-CoV-2 recognition}

In SARS-CoV, receptor recognition and membrane fusion occur through the viral S glycoprotein, which is present on the surface. As soon as the virus enters the host, the trimeric $S$ protein is split into two subunits, S1 and S2, followed by viral release of the S1 subunit for post-fusion confirmation. Subsequently, the S1 subunit binds with the peptidase domain of ACE2 via its $\mathrm{RBD}$, whereas membrane fusion takes place via the $\mathrm{S} 2$ subunit. Once the S1 subunit binds to the ACE2 peptidase domain, a single cleavage site is exposed on $\mathrm{S} 2$, which is subsequently sliced by various host proteases. This mechanism is crucial for viral infection. The SARS-CoV-2 S protein can also take advantage of ACE2 for host infection in a similar manner.

ACE2 also serves as a chaperone for membrane trafficking of the AA transporter B0 AT1(SLC6A19). B0 AT1 is involved in sodium-dependent uptake of neutral $A A$ in intestinal cells. With the stabilization of BO AT1, the comprehensive structure of ACE2 in a dimeric assembly has been reported by high-resolution studies [23]. Docking of the S protein trimer onto the structure of the ACE2 dimer together with the RBD of the $S$ protein revealed that two $S$ protein trimers bind to an ACE2 dimer simultaneously. Using a structure-based rational strategy, neutralizing antibodies or decoy ligands with greater affinities for either coronavirus S protein or ACE2 could be produced for viral infection treatment modalities [23].

\section{ACE2 variants and their association with SARS-CoV-2}

Recently, it was suggested that ACE2 variants affect ACE2 levels in humans. To validate this, the Leeds Family Study estimated ACE, ACE2, and neutral endopeptidase plasma levels in 534 individuals. It was predicted that various genetic profiles could account for up to $67 \%$ of the phenotypic variation in circulating ACE2 [32]. Of the various ACE2 variants, rs2106809 was hypothesized to primarily affect the circulating ACE2 levels that are comparatively higher in the CC or CT genotype than in the TT genotype. Moreover, microRNAs (miRNAs) can act as intermediaries in potential pathways that can regulate endothelial activities through posttranscriptional degradation and/or translational repression [33]. Important variations in the intermolecular interaction between the SARS-CoV-2 S protein and ACE2 alleles have been elucidated by molecular superimposition and comparative modeling. Of note, the ACE2 alleles, rs73635825 (S19P) and rs143936283 (E329G), produce proteins demonstrating weaker binding affinity and missing key residues for SARS-CoV-2 S protein complex formation. These findings might indicate an intrinsic resistance mechanism to SARS-CoV-2 infection [34]. Thus, these alleles might undergo positive selection under new selection pressure provided by SARS-CoV-2. However, as protein-protein and ligand-protein interactions are vital, molecular dynamic simulation could be used to prove an important interlink between various ACE2 alleles and the SARS-CoV-2 $S$ protein. Thus, analytical means for predicting the binding affinity of intermolecular complexes may offer valuable insights. Furthermore, the correlation between viral binding and ACE2 variants in patients could help identify various mechanisms underlying the pathophysiology and severity of COVID-19.

\section{ACE2 expression in individuals across different ethnicities}

ACE2 expression at cellular levels differs among individuals. Both ACE2 variants and their expression might affect individual susceptibility to SARS-CoV-2 infection and disease severity [14]. This information might provide a means for controlling viral infection by suppressing ACE2 expression levels in cells. COVID-19 results in progressive respiratory failure and sometimes even death owing to alveolar lung damage. The binding affinity of SARS-CoV-2 to ACE2 is 10-20-fold higher than that of SARSCoV [35]. Recent epidemiological studies on COVID-19 positive cases from China have reported that the overall incidence of COVID-19 is marginally higher in males; however, the proportion of males far exceeds that of females among chronically ill and fatal cases. Individuals over 60 years of age or those with chronic illnesses, such as hypertension and type 2 diabetes mellitus, 
are often associated with systemic inflammation or cytokine storm. Variations in immunity and ACE2 expression may lead to specific vulnerability and intensity of SARS-CoV-2 infection [36, 37].

In East Asia, the viral infection has spread exponentially; older males, in particular, are more affected by SARS-CoV and the recent SARS-CoV-2 infection. ACE2 expression, which varies between males and females, also differs between the world's three main racial groups: African, Asian, and Caucasian. Recently, Chen, et al. [38] reported higher expression of ACE2 in some organs, such as the adrenal gland, adipose tissue, esophagus, and heart, of Asian females than that in males. Expression levels also vary among other ethnic groups. Expression levels are moderately increased in the lungs, blood vessels, muscles, and ovaries. Typically, ACE2 expression decreases with age in both sexes and all ethnic groups. However, significant findings were only detected in Caucasian group, as the sample size was comparatively larger. Another important finding was that males had a more significant age-dependent decrease compared with females. For example, in the Caucasian group, few females demonstrated decreased ACE2 expression with increasing age in the blood, colon, and adrenal gland. However, in males, the age dependent decrease in ACE2 expression was observed in the blood, colon, adrenal gland, nerves, adipose tissue, and salivary glands [38]. The patterns of ACE2 expression found in this study were entirely different from those revealed by other studies projecting the inductive effect of increased ACE2 expression in the population on SARS-CoV-2 virus infection susceptibility and associated fatality.

Consistently, the prevalence of expression quantitative trait loci (eQTL) that show higher ACE2 expression is nearly $100 \%$ in East Asians and $>30 \%$ in other racial groups. ACE2 expression at the molecular level depends on many factors such as the positive impact of estrogen and androgen levels (both decrease with age) and the binding of virus infection-related transcription factors at ACE2 regulatory regions [39]. In addition, type 2 diabetes mellitus and inflammatory cytokines are involved in the repression of $A C E 2$ expression.

ACE2 expression analyses of the lung tissues of Asian and Caucasian group remain controversial. The ACE2 expressing cell ratio, detected using single-cell RNA-seq analysis, indicated that Asian donors had significantly increased ACE2 expression compared with African-American and Caucasian donors [40]. However, no differential expression of ACE2 has been observed between males and females or between younger and older individuals in normal human tissues [41]. As ACE2-expressing cells comprise a minor proportion of lung tissue cells [40], these conclusions may have been influenced by the purity of the ACE2 positive cells and sample size. Cao, et al. [39] reported that the heterogeneity in ACE2 expression among different ethnic groups can be explained by variations in the distribution and allele frequencies of ACE2 eQTLs in divergent populations. In addition, the average difference in allele frequencies of eQTLs between South Asian and East Asians has suggested a potential deviation in ACE2 expression in various populations and ethnicities in Asia. Table 1 summarizes the studies that have assessed ACE2 variants and expression in association with susceptibility and disease severity.

\section{ACE2 expression associated with age and SARS-CoV infection}

A different trend of ACE2 expression has been observed in children. In general, children with confirmed COVID-19 were mildly symptomatic or mostly asymptomatic. Children usually have higher plasma ACE2 levels than adults [42]. For example, ACE2 plasma levels in children (six months to 17 years of age) were 13-100 U/L compared with 9-67 U/L in the adult population (using a furanacryloyl-L-phenylalanylglycylglycine-based enzymatic activity assay) [33]. Fewer COVID-19 cases have been reported in children than in higher age groups. In a study including 1,099 patients in China, only $0.9 \%$ of the confirmed cases were under nine yrs of age and only $1.2 \%$ were $10-19$ years old [37]. This phenomenon agrees with a previously reported mouse study, in which younger animals were found to be resistant to SARS-CoV infection [43]. The correlation between COVID-19 severity and age can be explained based on immune decline (immune-senescence), as well as an unusual child-specific plasma ACE2 profile. However, ACE can traverse the placenta; it helps the mother pass on her immunity and other serum antibodies to the baby. During the second and third trimester of pregnancy, ACE2 plasma and urine levels were found to be elevated along with an increase in local placental/uterine output and ACE2 functions. This explains the systemic hemodynamic role in placental-fetal blood flow increase and rapid growth of the fetus [44].

\section{Transmembrane protease serine 2}

In humans, the TMPRSS2 gene encodes transmembrane protease, serine 2 (TMPRSS2), an enzyme belonging to the serine protease family. This protein contains a type 2 transmembrane domain along with a receptor class A domain. In addition, a scavenger receptor cysteine-rich domain and a protease do- 


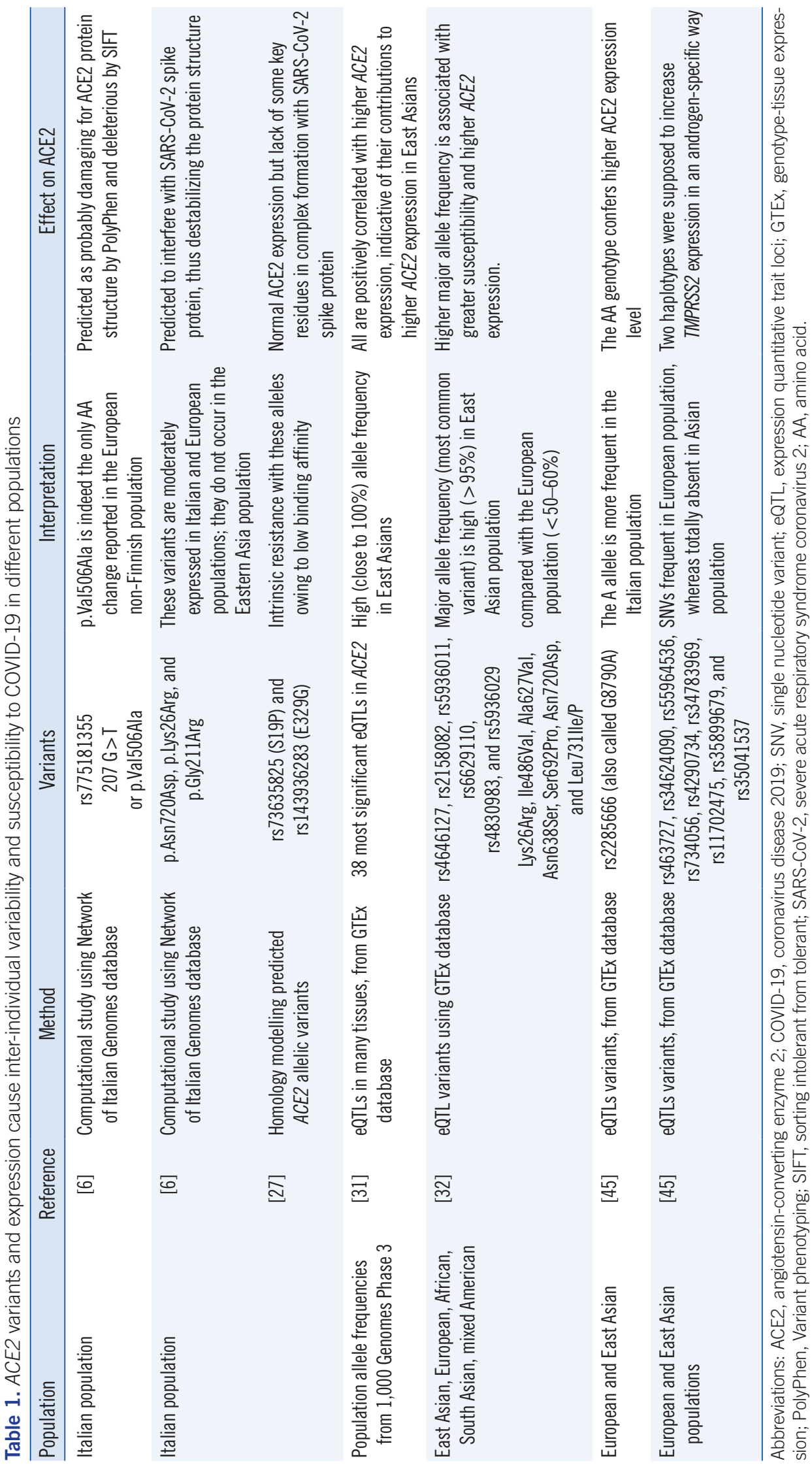


main have also been identified [45]. These proteases are involved in several pathological and physiological processes that take place in the human body. TMPRSS2 has been shown to be upregulated by androgenic hormones in prostate cancer cells; however, in androgen-independent prostate cancer, it was found to be downregulated [46].

TMPRSS2 can also cleave and activate the SARS-CoV-2 S protein during membrane fusion. This protease cleaves the SARS-CoV-2 receptor, the ACE2 carboxypeptidase, and ACE2 cleavage has been shown to augment viral entry into the various host cells [47]. TMPRSS2 also serves as an activator of SARSCoV entry into the host cells via the same mechanism. It is mostly expressed in the epithelial cells of human lungs, absorptive enterocytes, and upper endothelial cells of the esophagus, and it activates influenza A virus and meta-pneumo virus in culture cells [48]. The virus replication stage at which proteases cleave the viral glycoproteins in the plasma membrane varies between SARS-CoV and other viruses such as meta-pneumo virus and influenza A virus [48]. In the influenza A virus and meta-pneumo virus, entry is enabled by TMPRSS2 making a simple cut in the cell membrane glycoprotein, whereas in SARSCoV-2, the S protein is cleaved after the receptor induces a few conformational changes in protease structure [49]. Initially, the protease cleavage site in the $\mathrm{S}$ protein was predicted to be exposed after receptor binding; however, it was found to be located very near the C-terminal region of S protein [50]. Further studies have reported that the $\mathrm{S}$ protein of mouse hepatitis virus type 2 (MHV-2) usually undergoes conformational changes in two phases, which are facilitated by progressive receptor binding and proteolysis, to be activated for fusion [51]. The MHV-2 $S$ protein was found to be similar to the SARS-CoV S protein. Fusion and effective cleavage, while the receptor binds to the target cells, is very tightly controlled. Premature proteolysis of the activating cleavage site is also prevented efficiently in this way [52].

Cellular factors required for SARS-CoV-2 entry might provide information regarding viral transmission. Thus, protease dependence of SARS-CoV-2 entry has been studied [47].

Endosome cysteine proteases cathepsin B/L (CatB/L) are employed for SARS-CoV-2 S protein priming in cells along with TMPRSS2 protease activity. Priming by TMPRSS2 is essential for viral entry and for further spread in the affected host, whereas CatB/L activity is non-essential. Various studies have indicated that SARS-CoV-2 infection rate is also affected by TMPRSS2 proteolytic activity, which raises questions regarding the residual $\mathrm{S}$ protein priming conducted by $\mathrm{CatB} / \mathrm{L}$. It is hypothe- sized that furin-mediated pre-cleavage at the S1/S2 site in virally infected cells allows protease dependent entry into these cells, similar to MERS-CoV [47]. In addition, TMPRSS2 is required for homeostasis maintenance and various developmental changes and thus constitutes a drug target for future use. Importantly, TMPRSS2 protease activity is blocked by the serine protease inhibitor camostat mesylate.

Human trials have been recently approved in Japan for testing potential anti-pancreatitis agents; however, the condition is not associated with viral infection. These compounds and various others have been found to significantly increase antiviral activity and thus might be considered as an effective off-label treatment option for SARS-CoV-2-induced respiratory distress [47]. A recent study has reported that using a purified soluble form of TMPRSS11a, receptor binding to the pseudo-typed SARS-CoV S protein significantly enhanced virus multiplication rate. In addition, proteolytic cleavage was shown to be similar to trypsin [53]. A specific spatial orientation of the TMPRSS2 protease with respect to the SARS-CoV-2 S protein is a major factor underlying receptor binding. Additionally, receptor-bound S protein interaction with TMPRSS2 occurs in a specific spatial orientation at the cell surface resulting in inefficient cleavage of SARS-CoV-2 S protein and subsequent membrane fusion. Arginine and lysine residues within ACE2 AAs 697-716 have been predicted to be of prime importance for TMPRSS2-mediated cleavage and histone acetyltransferase activity. In addition, ACE2 needs to be processed for SARS-S-driven entry mediated by these proteases. TMPRSS2 increases SARS-S-entry, because it competes with metalloprotease A disintegrin and metalloprotease 17 for ACE2 processing [54].

\section{TMPRSS2 variants}

TMPRSS2 variants have been postulated to account for SARSCoV-2 epidemiological data. Recently, allele frequency differences of rs12329760 (a known TMPRSS2 variant) and eQTL haplotypes (genetic loci that explain genetic variations in mRNA expression levels) were reviewed in Italian and East Asian populations [55]. In the Italian population, death rates and variation in COVID-19 severity in both sexes were significantly higher than those in the East Asian population. Furthermore, the Italian population demonstrated a remarkable decrease in the load of detrimental TMPRSS2 protease variants compared with other Europeans, suggesting augmented TMPRSS2 protein/activity. Thus, TMPRSS2 plays an important role in disease severity. The catalytic triad of TMPRSS2 comprises a set of three coordinated AA, H296, D345, and S441, found at the active site of prote- 
ases; this special catalytic pocket structure helps in the hydrolysis of the highly specific substrate protein. Comparison between the Italian and East Asian (also Europeans) populations showed that four variants exhibited significantly distinct frequencies. Three of these single nucleotide variants (SNVs) are synonymous, and one is a missense substitution, p.Val160Met. Because of this substitution, the serine protease catalytic triad is spared, and the effect takes place on a residue away from cataIytic triad. Initially, this TMPRSS2 protease variant was shown to have functional links with the risk of prostate cancer [56]. In terms of eQTLs, several variants demonstrating a significant effect on TMPRSS2 expression in the lung epithelial tissue [genotype-tissue expression (GTEx) data] have been reported in the 3' region of the gene sequence [55].

In particular, two different haplotypes can be inferred from the GTEx frequency data [55]:

the haplotype that is more regularly seen is the "European" haplotype that comprises SNVs rs463727, rs34624090, rs55964536, rs734056, rs4290734, rs34783969, rs11702475, rs35899679, and rs35041537. This haplotype was found to be co-expressed and co-regulated with an eQTL (rs8134378). The eQTL (rs8134378) for TMPRSS2 is located $13 \mathrm{~kb}$ upstream of an androgen responsive enhancer, possibly upregulating the gene in an androgen-specific manner [57]. However, this haplotype was missing in the Asian population.

Three SNVs, rs2070788, rs9974589, and rs7364083, comprise another haplotype, which was found to have increased TMPRSS2 expression. Compared with East Asians, the Italian population had a $9 \%$ increase in this minor allele frequency [55].

\section{Dipeptidyl peptidase-4 (DPP4)}

DPP4 is a transmembrane glycoprotein. It is an ecto-peptidase that cleaves amino-terminal dipeptides causing $T$ cell activation and hence functions in host cell immune-regulation against viral infections. It also functions as a binding protein and ligand of extracellular factors like collagen and fibronectin. DPP4 is expressed by the epithelial and endothelial cells of vessels, as well as by the kidneys, intestines, lungs, and smooth muscle cells of the vasculature. DPP4 accelerates lung inflammation and causes fatal respiratory distress in MERS-CoV infection. Both SARS-CoV-2 and MERS-CoV affect the lower respiratory tract and cause ARDS, thus suggesting a link between SARS-CoV-2 and DPP4.

Molecular interactions between SARS-CoV-2 and DPP4 have been studied via computational model-based docking and 3D structures of the SARS-CoV-2 S glycoprotein and human DPP4 [58]. This model suggests a tight interaction between the S glycoprotein domains and DPP4 receptor. DPP4 binding residues K267, T288, A289, A291, L294, I295, R317, Y322, and D542 interact with the MERS-CoV S protein. The SARS-CoV-2 S1 domain has shown a similar -interaction with DPP4 suggesting that MERS-CoV and SARS-CoV-2 share similar pathways while interacting with host cells. Thus, DPP4 inhibitors, such as gliptins, could be used in COVID-19 patients to significantly reduce viral infection and multiplication rate, as well as reduce cytokine storm and inflammation in the lower airways [59].

\section{Epigenetic aspects of immune response in SARS-CoV-2 infection}

Macrophages and dendritic cells constitute key "hazard" signals among various cells of the innate immune system. These signals are stimulus-specific and cell-specific to ensure the onset of spatial and temporal responses. Such cell-specific signals are facilitated by cytokine, interleukin, and chemokine secretion or direct cell-cell communication. Consequently, their epigenetic ability to alter within minutes of a stimulus is not only important for rapid antiviral host response activation, but also necessary to maintain a sustained and specific defense response. It has been suggested that epigenetic regulation is accountable for virus entry priming and retention of this highly regulated host immune response over the initial activation wave [60].

Previous studies have focused on epigenetic aspects of the activation and formation of the innate and adaptive immune responses. The primary responsibility of the innate system is manifested via interferon and tumor necrosis factor genes. Interferons are effective mediators in preventing viruses from entering and activating the pathogenic immune response [61, 62]. Consequently, many viruses have possibly developed virulent mechanisms to counteract specific interferon-stimulated gene effectors [63]. Interferons and immune responses are sensitive to epigenetic modifications through different epigenetic pathways [64]. In contrast, interferon-stimulated genes generally show lower amounts of inducing histones, such as H3K4me3 and $\mathrm{H} 4 \mathrm{Ac}$, and lower RNA polymerase II occupancy [65]. Further chromatin remodelers and transcription factors are needed for the initiation of transcription at these genes including ATPdependent chromatin remodeling complex SWItch / sucrose non-fermentable (SWI / SNF) [66, 67].

Viruses have also developed various pathways to antagonize and disrupt epigenetic regulatory mechanisms, such as viral protein interference with host histone modification enzymes 
[68], chromatin remodeling machinery, and modified histones [61]. Current epigenetic studies have focused on the effects of genetic processes on DNA, rather than emphasizing the role of the genetic code itself. Major areas of focus include chromatin remodeling, histone methylation, and many other processes that affect how transcription is initiated, as well as how DNA is packaged in cells. Recently, Pinto et al. have reported that histone modification (H3K4me1, H3K4me3, H3K27Ac) in ACE2 gene is associated with increased ACE2 expression and they have also mentioned upregulation of NAD-dependent histone deacetylase Sirtuin 1 (SIRT1) is associated with increased ACE2 expression [69]. Similarly, Sawalha et al. have observed that decreased DNA methylation of ACE2 promoter (hypomethylation) was associated with increased ACE2 expression [70].

Corley, et al. [69] analyzed public datasets and revealed that DNA methylation levels associated with the ACE2 gene varied by tissue type, sex, and lung disease, as well as biological age in airway epithelial cells. In particular, the significant relationship between ACE2 DNA methylation and biological age indicates that age-dependent differences in host response may be mediated by aging-associated ACE2 dysregulation and increased ACE2 expression. These ACE2 gene-associated DNA methylation findings convincingly indicate that ACE2 is regulated transcriptionally, post-transcriptionally, and post-translationally within the body [69].

Approximately $60 \%$ of mammalian genes are thought to be influenced by miRNAs, especially cancer, metabolism, development, and apoptosis regulatory pathways. Since the first viral miRNAs were discovered in the human Epstein-Barr virus (EBV) [71], nearly 320 viral miRNA precursors have been identified. Most of the characteristics of miRNA-regulated gene expression appear to be particularly beneficial for viruses. For example, viruses targeting specific human genes via viral miRNAs create a suitable environment for virus replication and survival. In addition, viral miRNAs constitute a potential escape from the host immune system, because the host itself produces the miRNAs in the same way. Recently, Saçar Demirci, et al. [72] identified miRNA-mediated interactions in SARS-CoV-2 infection by computational analysis. Interestingly, the viral miRNAs appear to target a total of 1,367 human genes, while 479 human miRNAs might target SARS-CoV-2 genes, which are mainly responsible for viral entrance, replication, biogenesis, and infection.

\section{CONCLUSIONS AND FUTURE PERSPECTIVES}

The rapid surge in COVID-19 cases and the lack of specific management guidelines to date highlight the urgency and importance of identifying genetic factors that may influence disease susceptibility and severity. At present, information regarding genetic variants of ACE2 and TMPRSS2 among various populations in association with SARS-CoV-2 infection rate and disease severity is limited. In addition, studies based on genomic data analysis from data banks need to be validated by experimental analysis. It has been reported that ACE2 expression varies in different ethnic populations with respect to the allele frequency of its variants, although different confounding factors, such as genetic variability, age-related variation, and other co-morbid factors, might be involved in disease progression. Thus, additional studies are imperative to gain a better understanding of the pathophysiology and the involvement of ACE2 variants, expression, and epigenetic aspects in COVID-19 patients.

\section{AUTHOR CONTRIBUTIONS}

SC and KS: Literature review and manuscript drafting and revision; PM: conceptualization and design, proofreading and editing, and critical revision; SM and PS: proofreading and supervision. All authors have read the manuscript and provided final approval.

\section{CONFLICTS OF INTEREST}

None declared.

\section{RESEARCH FUNDING}

None declared.

\section{ORCID}

Sarita Choudhary https://orcid.org/0000-0001-5168-3305

Karli Sreenivasulu https://orcid.org/0000-0001-7983-8313

Prasenjit Mitra https://orcid.org/0000-0003-4826-1587

Sanjeev Misra https://orcid.org/0000-0002-0641-0946

Praveen Sharma https://orcid.org/0000-0002-8324-737X

\section{REFERENCES}

1. Hong KH, Lee SW, Kim TS, Huh HJ, Lee J, Kim SY et al. Guidelines for laboratory diagnosis of coronavirus disease 2019 (COVID-19) in Korea. Ann Lab Med 2020;40:351-60.

2. Mitra P, Misra S, Sharma P. COVID-19 pandemic in India: what lies 
ahead. Ind J Clin Biochem 2020;35:257-9.

3. Sung $\mathrm{H}$, Roh KH, Hong KH, Seong MW, Ryoo N, Kim HS et al. COVID-19 molecular testing in Korea: practical essentials and answers from experts based on experiences of emergency use authorization assays. Ann Lab Med 2020;40:439-47.

4. Mitra P, Suri S, Goyal T, Misra R, Singh K, Garg MK, et al. Association of comorbidities with coronavirus disease 2019: a review. Ann Natl Acad Med Sci (India) 2020;56:102-11.

5. WHO. Coronavirus disease (COVID-19) dashboard. https://covid19.who. int (Updated on Jul 2020).

6. Lingeswaran M, Goyal T, Ghosh R, Suri S, Mitra P, Misra S, et al. Inflammation, immunity and immunogenetics in COVID-19: a narrative review. Indian J Clin Biochem 2020;35:260-73.

7. Lacoma A, Mateo L, Blanco I, Méndez MJ, Rodrigo C, Latorre I, et al. Impact of host genetics and biological response modifiers on respiratory tract infections. Front Immunol 2019;10:1013.

8. Benetti E, Tita R, Spiga O, Ciolfi A, Birolo G, Bruselles A, et al. ACE2 gene variants may underlie interindividual variability and susceptibility to COVID-19 in the Italian population. Eur J Hum Genet 2020:1-13.

9. Chen YY, Zhang P, Zhou XM, Liu D, Zhong JC, Zhang CJ, et al. Relationship between genetic variants of ACE2 gene and circulating levels of ACE2 and its metabolites. J Clin Pharm Ther 2018;43:189-95.

10. Patel SK, Wai B, Ord M, Maclsaac RJ, Grant S, Velkoska E, et al. Association of ACE2 genetic variants with blood pressure, left ventricular mass, and cardiac function in Caucasians with type 2 diabetes. Am J Hypertens 2012;25:216-22.

11. Zhang SF, Tuo JL, Huang XB, Zhu X, Zhang DM, Zhou K, et al. Epidemiology characteristics of human coronaviruses in patients with respiratory infection symptoms and phylogenetic analysis of HCoV-OC43 during 2010-2015 in Guangzhou. PLoS One 2018;13:e0191789.

12. Hu B, Ge X, Wang LF, Shi Z. Bat origin of human coronaviruses. Virol J 2015;12:221.

13. Pyrc K, Berkhout B, van der Hoek L. The novel human coronaviruses NL63 and HKU1. J Virol 2007;81:3051-7.

14. Devaux CA, Rolain JM, Raoult D. ACE2 receptor polymorphism: susceptibility to SARS-CoV-2, hypertension, multi-organ failure, and COVID-19 disease outcome. J Microbiol Immunol Infect 2020;53:425-35.

15. Ye J, Zhang B, Xu J, Chang Q, McNutt MA, Korteweg C, et al. Molecular pathology in the lungs of severe acute respiratory syndrome patients. Am J Pathol 2007;170:538-45.

16. To KF, Tong JH, Chan PK, Au FW, Chim SS, Chan KC, et al. Tissue and cellular tropism of the coronavirus associated with severe acute respiratory syndrome: an in-situ hybridization study of fatal cases. J Pathol 2004;202:157-63.

17. Nicholls JM, Poon LL, Lee KC, Ng WF, Lai ST, Leung CY, et al. Lung pathology of fatal severe acute respiratory syndrome. Lancet 2003;361: 1773-8.

18. Hamming I, Timens W, Bulthuis ML, Lely AT, Navis G, van Goor H. Tissue distribution of ACE2 protein, the functional receptor for SARS coronavirus. A first step in understanding SARS pathogenesis. J Pathol 2004;203:631-7.

19. Tipnis SR, Hooper NM, Hyde R, Karran E, Christie G, Turner AJ. A human homolog of angiotensin-converting enzyme. Cloning and functional expression as a captopril-insensitive carboxypeptidase. J Biol Chem 2000;275:33238-43

20. Patel VB, Zhong JC, Grant MB, Oudit GY. Role of the ACE2/angiotensin $1-7$ axis of the renin-angiotensin system in heart failure. Circ Res 2016;118:1313-26.

21. Vickers C, Hales P, Kaushik V, Dick L, Gavin J, Tang J, et al. Hydrolysis of biological peptides by human angiotensin-converting enzyme-related carboxypeptidase. J Biol Chem 2002;277:14838-43.

22. Gheblawi M, Wang K, Viveiros A, Nguyen Q, Zhong JC, Turner AJ, et al. Angiotensin converting Enzyme 2: SARS-CoV-2 receptor and regulator of the renin-angiotensin system: celebrating the 20th anniversary of the discovery of ACE2. Circ Res 2020;126:1456-74.

23. Yan R, Zhang Y, Li Y, Xia L, Guo Y, Zhou Q. Structural basis for the recognition of SARS-CoV-2 by full-length human ACE2. Science 2020;367: 1444-8.

24. Li W, Moore MJ, Vasilieva N, Sui J, Wong SK, Berne MA, et al. Angiotensin-converting enzyme 2 is a functional receptor for the SARS coronavirus. Nature 2003;426:450-4.

25. Walls AC, Park YJ, Tortorici MA, Wall A, McGuire AT, Veesler D. Structure, function, and antigenicity of the SARS-CoV-2 spike glycoprotein. Cell 2020;181:281-92.e6.

26. Fu J, Zhou B, Zhang L, Balaji KS, Wei C, Liu X, et al. Expressions and significances of the angiotensin-converting enzyme 2 gene, the receptor of SARS-CoV-2 for COVID-19. Mol Biol Rep 2020;47:4383-92.

27. Chen Y, Guo Y, Pan Y, Zhao ZJ. Structure analysis of the receptor binding of 2019-nCoV. Biochem Biophys Res Commun 2020;525:135-40.

28. Zhou P, Yang XL, Wang XG, Hu B, Zhang L, Zhang W, et al. A pneumonia outbreak associated with a new coronavirus of probable bat origin. Nature 2020;579:270-3.

29. Qiu Y, Zhao YB, Wang Q, Li JY, Zhou ZJ, Liao CH, et al. Predicting the angiotensin converting enzyme 2 (ACE2) utilizing capability as the receptor of SARS-CoV-2. Microbes Infect 2020;22:221-5.

30. Li W, Zhang C, Sui J, Kuhn JH, Moore MJ, Luo S, et al. Receptor and viral determinants of SARS-coronavirus adaptation to human ACE2. EMBO J 2005;24:1634-43.

31. Wan Y, Shang J, Graham R, Baric RS, Li F. Receptor recognition by the novel coronavirus from Wuhan: an analysis based on decade-long structural studies of SARS coronavirus. J Virol 2020;94:e00127-20.

32. Rice GI, Jones AL, Grant PJ, Carter AM, Turner AJ, Hooper NM. Circulating activities of angiotensin-converting enzyme, its homolog, angiotensin-converting enzyme 2 , and neprilysin in a family study. Hypertension 2006;48:914-20.

33. Ciaglia E, Vecchione C, Puca AA. COVID-19 infection and circulating ACE2 levels: protective role in women and children. Front Pediatr 2020; 8:206.

34. Hussain M, Jabeen N, Raza F, Shabbir S, Baig AA, Amanullah A, et al. Structural variations in human ACE2 may influence its binding with SARS-CoV-2 spike protein. J Med Virol 2020:10.1002/jmv.25832.

35. Xia S, Liu M, Wang C, Xu W, Lan Q, Feng S, et al. Inhibition of SARSCoV-2 (previously 2019-nCoV) infection by a highly potent pan-coronavirus fusion inhibitor targeting its spike protein that harbors a high capacity to mediate membrane fusion. Cell Res 2020;30:343-55.

36. Wang D, Hu B, Hu C, Zhu F, Liu X, Zhang J, et al. Clinical characteristics of 138 hospitalized patients with 2019 novel coronavirus-infected pneumonia in Wuhan, China. JAMA 2020;323:1061-9.

37. Guan WJ, Ni ZY, Hu Y, Liang WH, Ou CQ, He JX, et al. Clinical characteristics of coronavirus disease 2019 in China. N Engl J Med 2020; 382:1708-20.

38. Chen J, Jiang Q, Xia X, Liu K, Yu Z, Tao W, et al. Individual variation of the SARS-CoV-2 receptor ACE2 gene expression and regulation. Aging Cell 2020;19:e13168.

39. Cao Y, Li L, Feng Z, Wan S, Huang P, Sun X, et al. Comparative genetic analysis of the novel coronavirus (2019-nCoV/SARS-CoV-2) receptor ACE2 in different populations. Cell Discov 2020;6:11.

40. Zhao Y, Zhao Z, Wang Y, Zhou Y, Ma Y, Zuo W. Single-cell RNA expression profiling of ACE2, the receptor of SARS-CoV-2. Am J Respir Crit Care Med 2020; 202:756-9. 
41. Li M, Li L, Zhang Y, Wang X. Expression of the SARS-CoV-2 cell receptor gene ACE2 in a wide variety of human tissues. Infect Dis Poverty 2020;9:45.

42. Bénéteau-Burnat B, Baudin B, Morgant G, Baumann FC, Giboudeau J. Serum angiotensin-converting enzyme in healthy and sarcoidotic children: comparison with the reference interval for adults. Clin Chem 1990;36:344-6.

43. Day CW, Baric R, Cai SX, Frieman M, Kumaki Y, Morrey JD, et al. A new mouse-adapted strain of SARS-CoV as a lethal model for evaluating antiviral agents in vitro and in vivo. Virology 2009;395:210-22.

44. Ghadhanfar E, Alsalem A, Al-Kandari S, Naser J, Babiker F, Al-Bader M. The role of ACE2, angiotensin-(1-7) and Mas1 receptor axis in glucocorticoid-induced intrauterine growth restriction. Reprod Biol Endocrinol 2017;15:97.

45. NCBI. TMPRSS2 transmembrane serine protease 2 [Homo sapiens (human)]. https://www.ncbi.nlm.nih.gov/gene/7113 [Updated on 20 Sep 2020].

46. Lucas JM, Heinlein C, Kim T, Hernandez SA, Malik MS, True LD, et al. The androgen-regulated protease TMPRSS2 activates a proteolytic cascade involving components of the tumor microenvironment and promotes prostate cancer metastasis. Cancer Discov 2014;4:1310-25.

47. Hoffmann M, Kleine-Weber H, Schroeder S, Krüger N, Herrler T, Erichsen S, et al. SARS-CoV-2 cell entry depends on ACE2 and TMPRSS2 and is blocked by a clinically proven protease inhibitor. Cell 2020;181: 271-80.e8.

48. Böttcher E, Matrosovich T, Beyerle M, Klenk HD, Garten W, Matrosovich M. Proteolytic activation of influenza viruses by serine proteases TMPRSS2 and HAT from human airway epithelium. J Virol 2006;80: 9896-8.

49. Shulla A, Heald-Sargent T, Subramanya G, Zhao J, Perlman S, Gallagher T. A. transmembrane serine protease is linked to the severe acute respiratory syndrome coronavirus receptor and activates virus entry. J Virol 2011;85:873-82

50. Watanabe R, Matsuyama S, Shirato K, Maejima M, Fukushi S, Morikawa $S$, et al. Entry from the cell surface of severe acute respiratory syndrome coronavirus with cleaved S protein as revealed by pseudotype virus bearing cleaved S protein. J Virol 2008;82:11985-91.

51. Belouzard S, Chu VC, Whittaker GR. Activation of the SARS coronavirus spike protein via sequential proteolytic cleavage at two distinct sites. Proc Natl Acad Sci 2009;106:5871-6.

52. Matsuyama S, Nagata N, Shirato K, Kawase M, Takeda M, Taguchi F. Efficient activation of the severe acute respiratory syndrome coronavirus spike protein by the transmembrane protease TMPRSS2. J Virol 2010; 84:12658-64.

53. Kam YW, Okumura Y, Kido H, Ng LFP, Bruzzone R, Altmeyer R. Cleavage of the SARS coronavirus spike glycoprotein by airway proteases enhances virus entry into human bronchial epithelial cells in vitro. PLoS One 2009;4:e7870.

54. Heurich A, Hofmann-Winkler H, Gierer S, Liepold T, Jahn O, Pöhlmann S. TMPRSS2 and ADAM17 cleave ACE2 differentially and only proteolysis by TMPRSS2 augments entry driven by the severe acute respiratory syndrome coronavirus spike protein. J Virol 2014;88:1293-307.

55. Asselta R, Paraboschi EM, Mantovani A, Duga S. ACE2 and TMPRSS2 variants and expression as candidates to sex and country differences in COVID-19 severity in Italy. Aging (Albany NY) 2020;12:10087-98.

56. FitzGerald LM, Agalliu I, Johnson K, Miller MA, Kwon EM, Hurtado-Coll A, et al. Association of TMPRSS2-ERG gene fusion with clinical characteristics and outcomes: results from a population-based study of prostate cancer. BMC Cancer 2008;8:230.

57. Clinckemalie L, Spans L, Dubois V, Laurent M, Helsen C, Joniau S, et al. Androgen regulation of the TMPRSS2 gene and the effect of a SNP in an androgen response element. Mol Endocrinol 2013;27:2028-40.

58. Senapati S, Kumar S, Singh AK, Banerjee P, Bhagavatula S. Assessment of risk conferred by coding and regulatory variations of TMPRSS2 and CD26 in susceptibility to SARS-CoV-2 infection in human. J Genet 2020;99:53

59. Vankadari $\mathrm{N}$ and Wilce JA. Emerging WuHan (COVID-19) coronavirus: glycan shield and structure prediction of spike glycoprotein and its interaction with human CD26. Emerg Microbes Infect 2020;9:601-4.

60. Beutler B. Innate immunity: an overview. Mol Immunol 2004;40:84559 .

61. Ivashkiv LB and Donlin LT. Regulation of type I interferon responses. Nat Rev Immunol 2014;14:36-49.

62. Schneider WM, Chevillotte MD, Rice CM. Interferon-stimulated genes: a complex web of host defenses. Annu Rev Immunol 2014;32:513-45.

63. García-Sastre A and Biron CA. Type 1 interferons and the virus-host relationship: a lesson in détente. Science 2006;312:879-82.

64. Stender JD and Glass CK. Epigenomic control of the innate immune response. Curr Opin Pharmacol 2013;13:582-7.

65. Agalioti T, Lomvardas S, Parekh B, Yie J, Maniatis T, Thanos D. Ordered recruitment of chromatin modifying and general transcription factors to the IFN- $\beta$ promoter. Cell 2000;103:667-78.

66. Busslinger M and Tarakhovsky A. Epigenetic control of immunity. Cold Spring Harb Perspect Biol 2014;6:a019307.

67. Smale ST, Tarakhovsky A, Natoli G. Chromatin contributions to the regulation of innate immunity. Annu Rev Immunol 2014;32:489-511.

68. Marban C, Suzanne S, Dequiedt F, de Walque S, Redel L, Van Lint C, et al. Recruitment of chromatin-modifying enzymes by CTIP2 promotes HIV-1 transcriptional silencing. EMBO J 2007;26:412-23.

69. Pinto BGG, Oliveira AER, Singh Y, Jimenez L, Gonçalves ANA, Ogava RLT, et al. ACE2 Expression is Increased in the Lungs of Patients with Comorbidities Associated with Severe COVID-19. J Infect Dis. 2020; 222:556-63.

70. Sawalha AH, Zhao M, Coit P, Lu Q. Epigenetic dysregulation of ACE2 and interferonregulated genes might suggest increased COVID-19 susceptibility and severity in lupus patients. Clin Immunol. 2020;215: 108410.

71. Pfeffer S, Zavolan M, Grässer FA, Chien M, Russo JJ, Ju J et al. Identification of virus-encoded microRNAs. Science 2004;304:734-6.

72. Saçar Demirci MD and Adan A. Computational analysis of microRNAmediated interactions in SARS-CoV-2 infection. PeerJ 2020;8:e9369. 